\title{
Triptolide induces mitochondrial apoptosis through modulating dual specificity phosphatase $1 /$ mitogen-activated protein kinases cascade in osteosarcoma cells
}

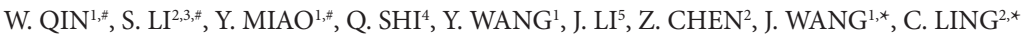 \\ ${ }^{1}$ Department of Medical Oncology, Changzheng Hospital, Second Military Medical University, Hetian Road, Shanghai, 200070, PR China; \\ ${ }^{2}$ Department of Traditional Chinese Medicine, Changhai Hospital, Second Military Medical University, Changhai Road, Shanghai, 200433, PR \\ China; ${ }^{3}$ Department of Endocrinology, Wuhan General Hospital of People's Liberation Army, Wuluo Road, Wuhan, 430070, PR China; ${ }^{4}$ Guang \\ An'men Hospital, China Academy of Chinese Medical Sciences, Beijing, Beixian'ge Road, 100053, PR China; ${ }^{5}$ General Hospital of Lanzhou Mili- \\ tary Command, West Xiaohu Road, Lanzhou, 31161962, PR China
}

*Correspondence: jiejunw19@163.com, lingchangquan@hotmail.com

"Contributed equally to this work.

Received January 9, 2017 / Accepted April 20, 2017

\begin{abstract}
Due to chemoresistance and metastasis, the overall prognosis of osteosarcoma (OS) has not improved over the last two decades. Exploring novel therapeutic agents that can circumvent theses malignant phenotypes of OS would be essential to improve the survival of OS patients. Triptolide is a unique diterpene triepoxide that possesses potent antitumor activities. However, the effects and mechanism of triptolide on OS cells remain unknown. The effects of triptolide on viability, apoptosis, cell cycle distribution and migratory ability of OS cells were measured using MTT, flow cytometry and wound healing and transwell invasion assays. And an OS tumor xenograft mouse model was produced to further study the in vivo antitumor effects of triptolide. The expression of DUSP1 at the protein and mRNA level in OS cells was detected by western blot and qPCR. We report that triptolide exhibits multidimensional antitumor activities in OS cells, including the induction of apoptosis and G1 phase accumulation, inhibition of cell viability, migration, and invasion. We further demonstrate that triptolide inhibits the expression of dual-specificity protein phosphatase1 (DUSP1) through inhibiting its promoter activity, which causes sustained activation of three subfamilies of mitogen-activated protein kinase (MAPK). And the modulation of DUSP1/MAPK cascade is associated with the apoptosis of OS cells, since the ectopic expression of DUSP1 or the inhibition of MAPK using specific inhibitors can counteract triptolide-induced apoptosis. In addition, triptolide enhances doxorubicin-induced apoptosis. In summary, our study suggests that DUSP1 is an important cellular target of triptolide, and triptolide may be a promising treatment option for OS as a single agent or combined with other chemotherapeutics.
\end{abstract}

Key words: triptolide, dual-specificity protein phosphatase 1, osteosarcoma, apoptosis, metastasis

Osteosarcoma (OS) is the most common malignant primary bone tumor accounting for approximately $15 \%$ of all primary bone tumors [1]. Despite the advances in aggressive chemotherapy, radiotherapy, and surgical resection, outcomes for newly diagnosed patients with OS had not changed in the past 20 years [2]. OS is generally fatal due to its acquired resistance to current therapeutic approaches and its tendency to metastasize to the lungs $[1,3]$. Thus, attempts to explore novel therapeutic agents or regimens that can overcome the resistant and metastatic phenotype of OS would be of great importance to improve survival of these patients.
MAP kinases (MAPKs) are widely expressed serine/ threonine kinases that are important in converting extracellular stimuli into a wide range of cellular responses, including cell proliferation, differentiation, and apoptosis [4]. MAPKs are composed of three major subfamilies, including c-Jun amino-terminal kinases (JNKs), extracellular-signal regulated kinases (ERKs), and P38s. MAPKs are often deregulated in cancers, and their isoforms function either as tumor suppressors or oncoproteins in different cellular contexts [5]. In general, ERKs exhibit pro-survival and growth-promoting activities in tumors, while p38s and JNKs are major pro-apoptotic mediators [5-7]. DUSP1, 
a member of the dual-specificity protein phosphatases (DUSPs) family, functions as a major negative regulator of MAPKs by specifically dephosphorylating and inactivating all three major subtypes of MAPKs [8]. The dysregulated DUSP1 has been widely implicated in many human cancers, although the precise role of this enzyme in tumorigenesis and tumor progression remains largely unexplored. DUSP1 is up-regulated in carcinomas derived from the colon, prostate, bladder, and liver, and siRNA silencing of endogenous DUSP1 resulted in inhibition of angiogenesis, invasion, and migration [9-11]. Furthermore, the up-regulation of DUSP1 positively correlates with resistance of cancer cells to chemotherapies which rely on JNK- or p38-induced apoptosis [10]. Accordingly, it has been reported that the up-regulated DUSP1 in OS cells correlates with cisplatin resistance, and inhibition of DUSP1 by siRNA silencing sensitizes OS cells to cisplatin-induced cell death. These results demonstrate that this enzyme may be a potential therapeutic target for the treatment of OS [12].

Triptolide is a structurally unique diterpene triepoxide isolated from Tripterygium wilfordii Hook F (TWHF), a Chinese medicinal plant used for treating a wide range of disorders for centuries [13-15]. Triptolide possesses a unique profile of biological activities, including anti-inflammatory, anti-rheumatic, immunosuppressive, and antitumor activities [13]. The antitumor effects of triptolide have been investigated in various tumor models in vitro and in vivo. Accumulated study data have demonstrated that triptolide possesses potent antitumor effects, as evidenced by its potent cytotoxicity, inducing apoptosis, circumventing drug resistance and inhibiting angiogenesis and metastasis in a number of cancer cell lines [16]. The mechanisms responsible for antitumor activities of triptolide have been extensively investigated in the past few years. Thus far, triptolide is generally considered as a multi-target natural chemotherapeutic compound, and a couple of signaling molecules, such as P53, NFkB, HSF-1, AP1, and RNA polymerase II have been proposed as the potential cellular targets of triptolide [13, 17]. Interestingly, it was demonstrated that triptolide potently blocked the expression of DUSP-1 induced by peptidoglycan and prolonged the activation of JNK and p38 in primary peritoneal macrophages cells [18]. However, to our best knowledge, the question of whether the triptolide-mediated inhibition of DUSP1 contributes to its antitumor activities remains controversial. In the present study, we demonstrated that DUSP1 is aberrantly up-regulated in multiple OS cells, and triptolide potently inhibits the expression of DUSP1, leading to sustained activation of MAPKs pathway. Moreover, the modulation of DUSP1/MAPKs cascade contributes to triptolide-induced apoptosis and enhances doxorubicin-mediated cell death. Our study suggests DUSP1 could be considered as a potential therapeutic target in OS therapy, and triptolide may be a promising treatment option for OS, as a single agent or combined with other chemotherapeutics.

\section{Materials and methods}

Cell lines, reagents and antibodies. Three OS cell lines (MG63, U2OS, UMR-106), and one osteoblast cell line (hFOB1.19) were obtained from Cell Bank of Type Culture Collection of the Chinese Academy of Sciences (Institute of Cell Biology, Chinese Academy of Sciences, Shanghai). All cell lines were grown in Dulbecco's Modified Eagle Medium (Gibco, NY) with 10\% fetal bovine serum (Gibco, $\mathrm{NY}$ ) and were maintained in an atmosphere of $5 \% \mathrm{CO} 2$ in a humidified $37^{\circ} \mathrm{C}$ incubator. Triptolide was purchased from Sigma (St. Louis, MO) and dissolved in Dimethyl Sulfoxide (DMSO) to obtain a $10 \mathrm{mM}$ stock solution. The ERK1/2 inhibitor PD98059, p38 inhibitor SB203580, JNK1/2 inhibitor SP600125 and pan-caspase inhibitor Z-VAD-FMK were obtained from Tocris (Bristol, UK). N-acetylcysteine (ROS scavenger) was from Sigma. Monoclonal antibodies to p-p53 (Ser-46), JNK1/2, p-JNK1/2 (Thr183/Tyr185), p38, p-p38 (Thr180/Tyr182), Erk1/2, p-Erk1/2 (Thr202/Tyr204), Bax, Bcl-2, Akt and p-Akt (Thr308) were purchased from Cell Signaling Technology (Beverly, MA); anti- $\beta$-actin and anti-p53 was from Sigma (St Louis, MO); anti-Cytochrome c, anti-DUSP-1, anti-cleaved caspase-3 and -9 were from Santa Cruz Biotechnology (Santa Cruz, CA).

Cell viability assays. MTT (3-(4, 5-dimethylthiazol-2-yl)2,5-diphenyltetrazolium bromide) assays were performed to determine the cell viability. Briefly, $1 \times 10^{4}$ cells per well were seeded into a 96-well plate and allowed to adhere overnight. After treatment with triptolide at various concentrations for 24 and $48 \mathrm{~h}$, MTT reagent was added into the fresh medium for formazan development. DMSO was used to dissolve the formazan crystals, after which the absorbance at $560 \mathrm{~nm}$ was measured using a microplate reader (Biotek, Winooski, VT). All experiments were done in quadruplicate and repeated at least three times.

Flow cytometry. OS cells $\left(1 \times 10^{5}\right.$ cells per well $)$ were seeded into a 6-well plate and allowed to adhere overnight. These cells were exposed to DMSO or $100 \mathrm{nM}$ triptolide for $12 \mathrm{~h}$, then the samples were collected and fixed in $70 \%$ ethanol overnight. For cell cycle analysis, fixed cells were treated with RNase for 20 minutes before addition of $5 \mathrm{mg} / \mathrm{mL}$ PI and analyzed by fluorescence-activated cell sorting (FACS). Cell apoptosis was measured by using fluorescein isothiocyanate (FITC) / propidium iodide (PI) double staining according to the manufacturer's instructions (KeyGEN, Nanjing, China). Cells were subjected to FACS assay and apoptotic cell death percentage represented FITC (+)/PI (-)-stained cells.

Immunofluorescence. For Hoechst33342 staining, cells were treated with DMSO or triptolide for $24 \mathrm{~h}$. The cells were fixed with paraformaldehyde (3.7\%) for $10 \mathrm{~min}$ at room temperature followed by washing twice with PBS. The cells were stained with $1 \mu \mathrm{M}$ Hoechst 33342 (Sigma, St Louis, MO) in PBS for $15 \mathrm{~min}$ at $37^{\circ} \mathrm{C}$. The unbound stain was removed by washing twice with PBS. Fluorescence images were collected using fluorescence microscope (Nikon, Tokyo, Japan). 
Migration and invasion assays. Cell migration (woundhealing) assay was conducted to examine the capacity of cell migration. The wound was generated by scratching the surface of the plates with a pipette tip. The cells were then incubated in the absence or presence of triptolide ( 25 $\mathrm{nM}$ ) for 48 hours and then photographed with an inverted microscope system (Leica DM IL, Wetzlar, Germany). Cell invasion assay was performed by using the transwell invasion chamber (Corning Costar, MA) coated with $30 \mu \mathrm{g}$ of Matrigel according to the vendor's protocol (BD, Bioscience, CA). The chamber is divided by a polycarbonate microporous filter (pore size $8 \mu \mathrm{m}$ ). Cells were placed in the upper chamber and cultured in Dulbecco's modified Eagle's medium (DMEM) containing $0.1 \%$ bovine serum albumin for $12 \mathrm{~h}$. DMEM containing 5\% FBS was added to the lower chambers as chemoattractant. Then culture media was gently replaced with fresh DMEM containing $25 \mathrm{nM}$ triptolide or vehicle control, and cells were incubated for additional $24 \mathrm{~h}$. Cells on the top surface of the filter were removed and cells that migrated to the bottom surface of the filter were fixed and stained by crystal violet in $2 \%$ ethanol for $4 \mathrm{~h}$ and then subjected to microscopic inspection. All experiments were conducted in triplicate and repeated twice.

Western blot. Total proteins were extracted with RIPA lysis buffer. The cytosolic fraction proteins were collected according to the manufacturer's directions (Beyotime, Haimen, China). Equal amounts of proteins were subjected to $10 \%$ sodium dodecyl sulfate-polyacrylamide gel electrophoresis (SDS-PAGE), and then transferred onto polyvinylidene fluoride membranes (Millipore, MA). The membranes were fixed and incubated with primary antibodies overnight at $4{ }^{\circ} \mathrm{C}$ with gentle agitation, then incubated with horseradish peroxidase-conjugated secondary antibody, and detected with enhanced chemiluminescence solution (Thermo Scientific, Rockford, IL).

Measurement of ROS production. Experiments were performed using the ROS assay kit (Beyotime, Haimen, China) according to the manufacturer's instructions. Briefly, cells seeded in 96-well plates were incubated with $100 \mu \mathrm{L} 1 \times$ dichlorofluorescin diacetate (DCFDA) solution at $37^{\circ} \mathrm{C}$ for 30 min and then washed with PBS three times in order to remove residual probes. The fluorescence was detected by FACS (BD, San Jose, CA). For each sample 10000 events were collected.

Quantitative real-time PCR (qRT-PCR). Total RNA was extracted using TRIzol reagent (Invitrogen, Carlsbad, CA). qRT-PCR was performed using an ABI 7300 Fast Real-Time PCR System (Applied Biosystems, Foster City, CA) and SYBR Green PCR kit (Takara, Otsu Shiga, Japan). Human mRNA reverse transcription-PCR primers were as follows: DUSP1, forward TGCAGTACCCCACTCTACGAT, and reverse AGGCATCCAGCATGTCCTTG; JNK, forward ACGCCTTATGTAGTGACTCG, and reverse CCCCACAGACCATAAATCCAC; p38, forward TGCGTCTGACAGGAACACCC, and reverse CGCAAAGTTCATCTTCGGCATC;
ERK, forward CCCCATCACAAGAAGACCTG, and reverse AGCCTGTTCTACTTCAATCCTCT; $\beta$-actin, forward GGCACCACCATGTACCCTG, and reverse CCGGACTCGTCATACTCCT.

Small interfering RNA (siRNA) synthesis. siRNA specific for the gene encoding DUSP-1 was bought from RiboBio (Guangzhou, China) and was a pool of three target-specific 19 25 nt siRNAs designed to knock down gene expression of DUSP-1. The scramble control siRNA (RiboBio) was used as the negative control. Transfections were performed using the Lipofectamine 2000 kit (Invitrogen, Carlsbad, CA) according to the manufacturer's instructions.

Vector construction. The complementary DNA (cDNA) encoding DUSP-1 was PCR-amplified by the Pfu Ultra II Fusion HS DNA Polymerase (Agilent Technologies, Palo Alto, CA), and was subcloned into the pcDNA3.1 vector (Invitrogen, Carlsbad, CA). Sequences of primers used in the amplification of the CDNA of DUSP-1 were CCCAAGCTTATGGTCATGGAAGTGGGCACCCT (forward) and GCTCTAGATCAGCAGCTGGGAGAGGTCGT (reverse). The DUSP-1 promoter luciferase reporter was constructed as previously described [19]. Briefly, the promoter region of DUSP1 was amplified by PCR using the following primers: CAAGTCTTCCGGGGGCCACAAGACTAGGAA (forward), and TCGCACACACAGCCCAAATGTCCTTCGCAG (reverse). The PCR product was ligated into the pGL3-basic reporter (Promega, Madison, WI), resulting in pGL3-DUSP1-luc.

Promoter cloning and dual-luciferase reporter (DLR) assay. U2OS cells seeded into 24 -well plates were co-transfected with $200 \mathrm{ng}$ of pGL3-DUSP1-luc and $40 \mathrm{ng}$ of pRL-TK vectors (Promega, Madison, WI) by Lipofectamine 2000 (Invitrogen, Carlsbad, CA) according to manufacturer's instructions. After $24 \mathrm{~h}$, cells were treated with triptolide at the indicated concentrations for additional $24 \mathrm{~h}$. Both, firefly and renilla luciferase activities in lysed cells, were measured using the Dual-Luciferase Reporter Assay System (Promega, Madison, WI) according to the manufacturer's instructions. The ratio of firefly and renilla luciferase activities represented the normalized firefly luciferase activity.

Osteosarcoma nude mice model. BALB/c nude mice, aged 4-5 weeks, were obtained from Shanghai Silaike Animal Experimental Center. All animal experiments were performed in accordance with the guidelines of the Second Military Medical University Animal Care Facility and our study followed the principles of the Declaration of Helsinki. To produce a xenograft model, $5 \times 10^{5} \mathrm{OS}$ cells per mouse were injected subcutaneously into the right flanks of nude mice. After growing for 7 days, mice were randomly divided into two treatment groups. Mice were given daily i.p. injections of triptolide $(0.2 \mathrm{mg} / \mathrm{kg})$ or vehicle (DMSO) for 10 days, respectively. To produce spontaneous metastasis models, $2 \times 10^{4}$ UMR-106 cells were inoculated intratibially as described previously [20]. Seven days later, mice were divided into two groups and treated as above mentioned. After anesthesia 
with ether and scarification, primary tumors were removed and weighed; the tumor volume $=\left(\right.$ length $\times$ width $\left.^{2}\right) / 2$. Lung metastases were counted under a dissecting microscope in a double blind setting. The sections of primary tumor tissue were fixed in formalin and embedded in paraffin for subsequent TUNEL or immunohistochemistry staining.

TUNEL assay. Paraffin-embedded tumor tissue sections from control and triptolide-treated mice were processed for the terminal deoxynucleotidyl transferase-mediated dUTP nick end labeling (TUNEL) assay according to the manufacturer's instructions (Chemicon, Temecula, CA). Briefly, the paraffin embedded tumor sections were dehydrated and cleared with xylene after hemotoxylin staining and then mounted with permount solution. The stained cells were counted under a microscope (Nikon, Tokyo, Japan).

Immunohistochemistry. The xenograft tumor slides were incubated with specific antibodies. Anti-rabbit or anti-mouse peroxidase-conjugated secondary antibodies (Cell Signaling Technology, Beverly, MA) were applied. Diaminobenzidine colorimetric reagent solution from Sigma (St Louis, MO) was used. Slides were counterstained with hematoxylin (Sigma Chemical Co). Staining of the whole tissue sections was examined and scored by 2 independent observers.

Statistical analysis. Experiments were repeated a minimum of three times. Data are expressed as mean $+/-$ SEM or mean +/- SD as indicated in the figure legends. Statistical significance of differences between means was determined by Student $t$ test or one-way analysis of variance (ANOVA). All p-values were obtained using the SPSS 16.0 software package (SPSS, Chicago, IL). A value of $\mathrm{p}<0.05$ was considered to be statistically significant.

\section{Results}

Effects of triptolide on viability, cell cycle distribution and migratory ability of OS cells. First, we examined the effect of triptolide on the cell viability of three OS cell lines with different p53 status. U2OS (p53 wild-type), MG63 (p53-null), and UMR-106 (p53 wild-type) cells were exposed to increasing concentrations of triptolide for $24 \mathrm{~h}$ or $48 \mathrm{~h}$, respectively. Then, the cell viability was measured using MTT cell viability assays. As shown in Figure $1 \mathrm{~B}$ and C, triptolide significantly decreased the cell viability of all three cell lines in a time- and dose-dependent manner, with $\mathrm{IC}_{50}$ values ranging from $259.8 \mathrm{nM}$ to $785.5 \mathrm{nM}$ at $24 \mathrm{~h}$ and $180.3 \mathrm{nM}$ to $255.6 \mathrm{nM}$ at $72 \mathrm{~h}$, respectively. Previous study showed that the functional p53 was required for the antitumor activity of triptolide [21]. However, in this study, we observed that triptolide also exhibited potent antitumor activity in p53-null MG63 cells. On the contrary, UMR-106 cells which express wild-type p53 were less sensitive than MG63 to the inhibitory effect of triptolide. Moreover, triptolide altered cell cycle distribution of OS cells. As shown in Figure 1E and F, triptolide induced significant G1 phase arrest in U2OS and MG63 cells. Lastly, the inhibitory effects of triptolide on migration and invasion abilities of OS cells were verified by wound healing and transwell invasion assays (Figure $1 \mathrm{G}$ and $\mathrm{H}$ ). Together, these results demonstrate that triptolide possesses potent and broad antitumor activities in OS cells, including the inhibition of cell viability, migration, invasion, and cell cycle progression.

Triptolide induced mitochondria-dependent apoptosis in OS cells. To elucidate the mechanism by which triptolide causes cell death in OS cells, we monitored triptolide-induced apoptosis using various methods. In Figure $2 \mathrm{~A}$, the induction of apoptosis was measured by flow cytometry and quantitatively expressed as the percentage of cells that were Annexin$\mathrm{V}$-positive and PI-negative. After $24 \mathrm{~h}$, triptolide dosedependently increased Annexin-V+/PI-population in both U2OS and MG63 cells, thus demonstrating the induction of apoptosis. Next, Hoechst-33342 fluorescent staining was performed to further confirm the induction of apoptosis. As shown in Figure 2B, typical apoptotic morphology, including nuclear condensation and the formation of apoptotic bodies, were observed in triptolide-treated cells. Mitochondria play an essential role in apoptosis triggered by chemical agents. The mitochondrial response includes the release of cytochrome $\mathrm{c}$ into the cytosol. Here we demonstrated that the intervention of triptolide markedly increased cytosolic concentration of cytochrome $c$, indicating the induction of mitochondrial apoptosis. Since high concentration of reactive oxygen species (ROS) can promote mitochondriadependent apoptosis [22], we wondered whether triptolide could affect the generation of ROS. As shown in Figure 2D, triptolide potently promoted ROS production in U2OS cells, which could be involved in triptolide-induced apoptosis.

To investigate the apoptotic pathway implicated, the activation of several caspases and the modulation of some signaling molecules were studied by western blot analysis. Treatment by triptolide for $24 \mathrm{~h}$ induces significant cleavage of caspases-3 and -9 in a dose-dependent manner (Figure 2E). Meanwhile, some proapoptotic proteins, including Bax and P53, were up-regulated by triptolide. Conversely, some anti-apoptotic molecules, such as Bcl-2 and Akt, were down-regulated. In addition, we also observed an enhanced phosphorylation of P53 and a decreased phosphorylation of Akt (Figure 2F and $2 \mathrm{G})$. Moreover, the use of the pan-caspase inhibitor Z-VADFMK (VAD) prevented apoptosis induced by triptolide, although this prevention was not total (Figure $2 \mathrm{H}$ ). All these results suggest that triptolide induces apoptosis in OS cells by a mechanism mainly dependent on activation of mitochondrial apoptotic pathway.

Triptolide suppressed exnograft tumor growth and lung metastasis. To further study the in vivo antitumor effects of triptolide, mice with OS tumor xenografts were treated with triptolide for 10 days. Administration of triptolide caused a $73.7 \%$ reduction of tumor volume (Figure $3 \mathrm{~A}$ and $\mathrm{B}$ ) and a $67.3 \%$ reduction of tumor weight (Figure 3C). Importantly, the number of lung metastases was also significantly decreased in triptolide-treated group (Figure 3D). The exten- 
A

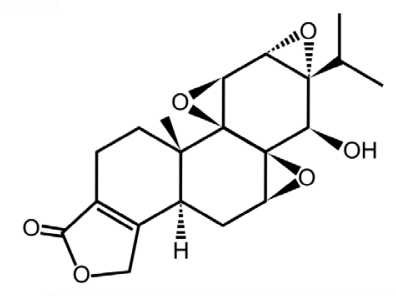

D
B

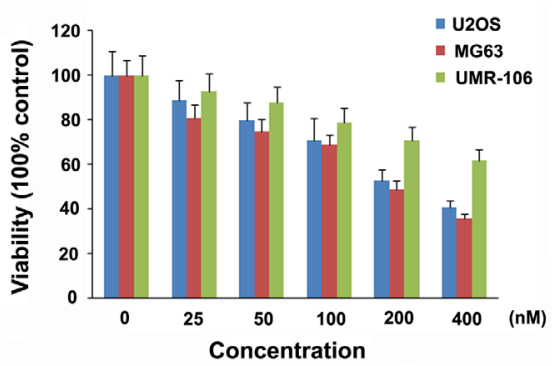

E

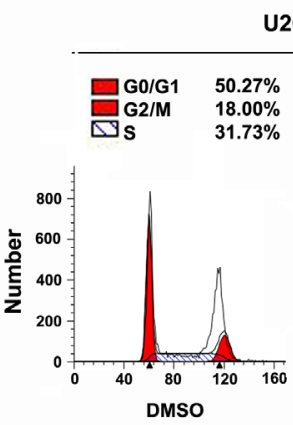

C

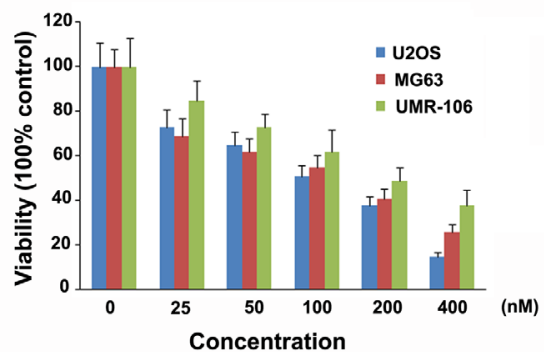

$\mathbf{F}$
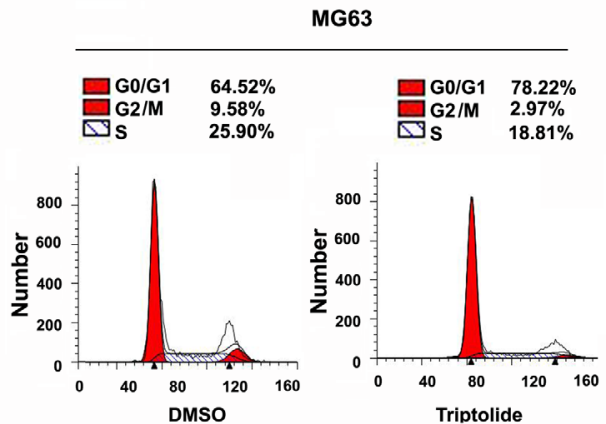

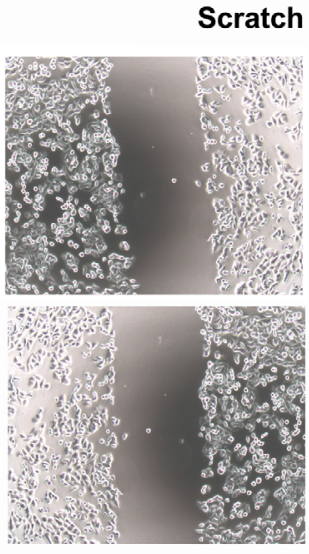

$\mathbf{O} \mathbf{h}$

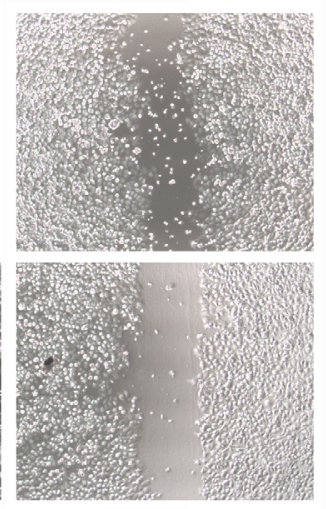

$48 \mathrm{~h}$
H

DMSO

Triptolide

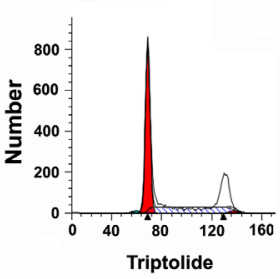

\section{Transwell assay}

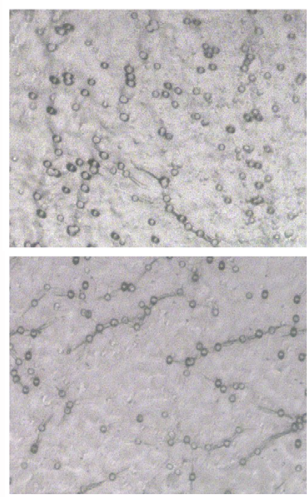

DMSO

Triptolide

Figure 1. The inhibitory effects of triptolide on viability, cell cycle progression and migratory abilities of osteosarcoma (OS) cells. (A) Molecular structure of triptolide. U2OS, MG63, and UMR-106 cells were exposed to indicated concentration of triptolide for $24 \mathrm{~h}$ (B) or $48 \mathrm{~h}$ (C); cell viability was analyzed by MTT dye assay and was normalized to the OD value of vehicle control. The values in the figures are expressed as the means \pm standard deviation (SD). (D) IC50 values of triptolide in U2OS, MG63, and UMR-106 cells at indicated time intervals. Each experiment was performed in quadruplicate. Data are mean. (E) U2OS cells and (F) MG63 cells were treated with DMSO or $100 \mathrm{nM}$ triptolide for $12 \mathrm{~h}$; cell cycle distribution was analyzed by PI staining. (G) Wound-healing and $(\mathrm{H})$ transwell assays were performed to confirm the effects of triptolide on cell migration and invasion, respectively. Original amplification, $100 \times$.

sive tumor cell apoptosis was evidenced by TUNEL and cleaved caspase-3 staining (Figure 3E). In addition, a lower PCNA staining was observed in triptolide treated group, suggesting that the proliferation activity in vivo was also inhibited by triptolide.
Triptolide inhibited the expression of DUSP1 through modulating its promoter activity and ROS production. We first examined the protein levels of DUSP1 in three OS cell lines. In Figure 4A, the protein expression of DUSP1 is up-regulated in all these OS cell lines as compared to normal 
A

$\overline{\mathbf{n}}$

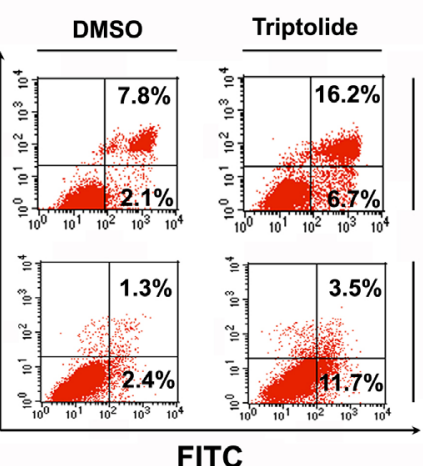

C

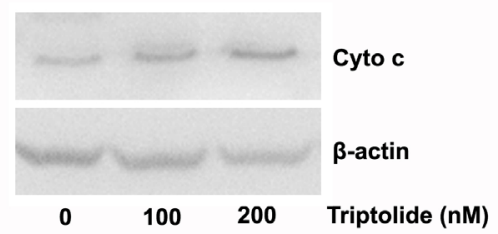

$\mathbf{E}$

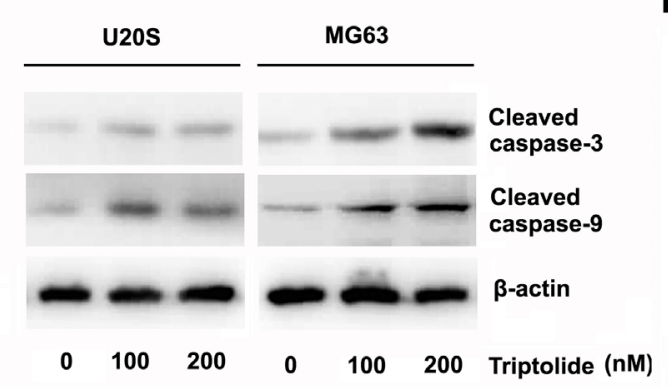

B

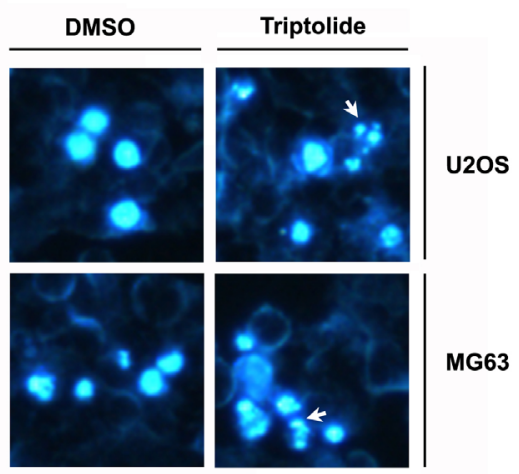

$\mathbf{G}$

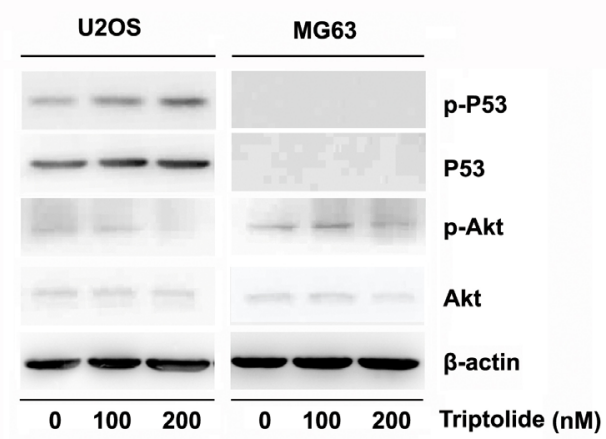

H

$\mathbf{F}$

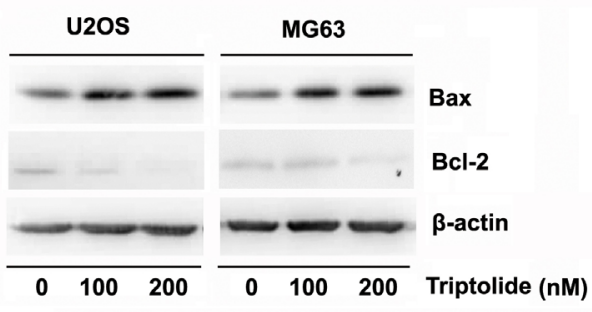

$\overline{\mathbf{n}}$

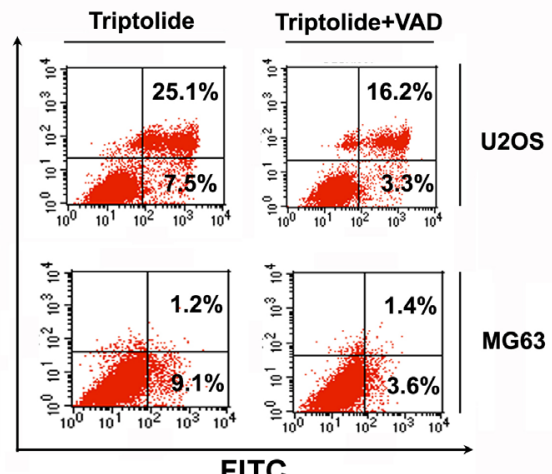

Figure 2. Triptolide induces apoptosis in OS cells with different P53 status. (A) U2OS (P53 wild type) and MG63 (P53 deleted) cells were treated with $200 \mathrm{nM}$ for $24 \mathrm{~h}$, the apoptotic cells were measured using FITC/PI double staining. Representative FACS data are shown. (B) Hoechst 33342 fluorescence staining was performed to further confirm the induction of apoptosis induced by triptolide. (C) Triptolide induces mitochondrial cytochrome $\mathrm{c}$ release. The cytosolic cytochrome $\mathrm{c}$ concentration was measured by western blot. (D) ROS (reactive oxygen species) production was augmented by triptolide. ROS generation was measured by DCFDA labeling. (E-G) Triptolide increases the expression of several pro-apoptotic proteins, including cleaved caspase-3 and -9, Bax, and P53, while decreases the expression of some anti-apoptotic proteins, such as Bcl-2 and Akt. The expression or phosphorylation of these molecules was measured by western blot. U2OS and MG63 cells were exposed to $200 \mathrm{nM}$ triptolide for $24 \mathrm{~h}$. Then total proteins were extracted and subjected to western blot analysis. (H) The effects of pan-caspase inhibitor Z-VAD-FMK (VAD) on triptolide-induced apoptosis in OS cells. Cell apoptosis was measured by flow cytometry. 
osteoblast cells hFOB1.19. We also observed that the protein expression of DUSP1 was increased after the treatment of subtoxic dose of doxorubicine, a commonly used chemotherapy drug of OS (Figure 4B). Consistent with previous studies showing that triptolide is a blocker of DUSP1 [23], we demonstrated that triptolide significantly inhibited the expression of DUSP1 both at the protein and mRNA level in OS cells (Figure 4C and D). Next, the DUSP1 promoter luciferase reporter assay was performed to determine whether triptolide down-regulated DUSP1 transcription through inhibiting its promoter activity. As shown in Figure 4E, the treatment by triptolide for $6 \mathrm{~h}$ caused a marked decrease of promoter activity of DUSP1. It was reported that ROS caused oxidation and inhibition of DUSP1 by converting their catalytic cysteine to sulfenic acid [24]. Therefore, we tried to determine whether the increase of ROS production is associated with triptolide-induced inhibition of DUSP1. In Figure $4 \mathrm{~F}$, the pretreatment of N-Acetylcysteine (NAC), a widely used ROS scavenger, partially blocked triptolideinduced inhibition of DUSP1 in U2OS cells, thus demonstrating that the increased generation of ROS also contributed to triptolide-induced inhibition of DUSP1. Taken together, these data demonstrate that DUSP1 is up-regulated in OS cells, and triptolide significantly suppresses the expression of this molecule through modulating its promoter activity and ROS generation.

The repression of DUSP1 contributed to triptolideinduced apoptosis and sensitizes OS cells to doxorubicinmediated cell death. We further investigated the biological effects of triptolide-induced inhibition of DUSP1 in OS cells using a combined overexpression and knockdown approach. In Figure 5A, knockdown of DUSP1 significantly enhanced triptolide-induced apoptosis in U2OS cells. Conversely, ectopic expression of DUSP1 antagonized the induction of apoptosis in triptolide-treated OS cells (Figure 5B). Consistent with our previous observations, IHC assays using antiDUSP1 antibody showed that triptolide-induced in vivo tumor growth inhibition was closely associated with its inhibition of DUSP1 (Figure 5C). Given that the up-regulation of DUSP1 is associated with chemoresistance in cancer cells, we wondered whether the treatment of triptolide could render OS cells more sensitivity to chemotherapeutics. Not surprisingly, the treatment of si-DUSP1 or triptolide significantly enhanced doxorubicine-induced apoptosis in U2OS cells (Figure $5 \mathrm{D}$ and E). Conversely, overexpression of DUSP1 partially reversed the synergistic effects between triptolide and doxorubicin (Figure 5F). In summary, the triptolide-induced inhibition of DUSP1 not only contributed to the induction of apoptosis in U2OS cells but also enhanced sensitivity of these cells to doxorubicine.

The DUSP1-dependent activation of MAPKs enhanced antitumor effect of triptolide. To further explore the mechanism underlying the DUSP1-dependent antitumor activities of triptolide, we measured the expression and phosphorylation of three major substrates of DUSP1, including ERK1/2,

\section{A}
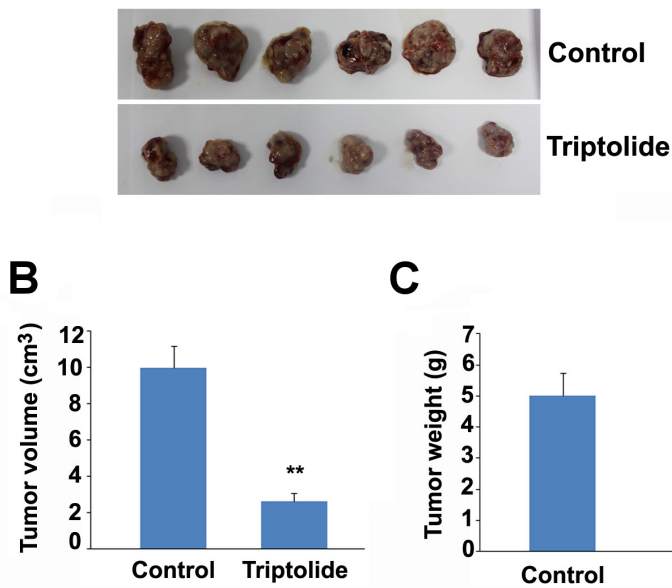

C

D

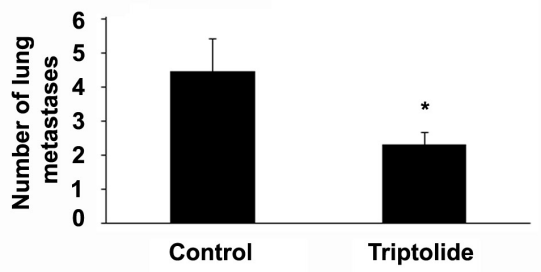

$\mathbf{E}$

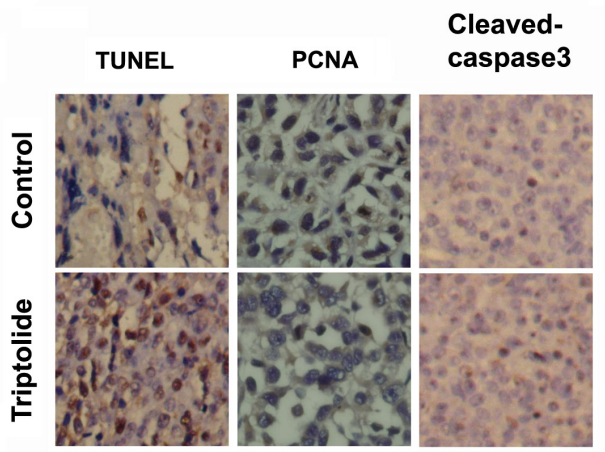

Figure 3. Triptolide inhibits tumor growth and lung metastases in OS xenograft mice model. Mice were given daily i.p. injections of triptolide $(0.2 \mathrm{mg} / \mathrm{kg} / \mathrm{day}$ or vehicle control (DMSO)) for 10 days. (A) Primary tumors removed from two treatment group of mice model. (B) Tumor volumes and $(\mathrm{C})$ tumor weights of triptolide-treated were significantly decreased, as compared to DMSO-treated group. (D) The treatment of triptolide suppresses lung metastasis in spontaneous metastatic mice model. Lung metastases were counted under a dissecting microscope in a double blind setting. ${ }^{*} \mathrm{p}<0.05,{ }^{* *} \mathrm{p}<0.01$. (E) TUNEL and IHC assays show the induction of apoptosis in planted tumors. TUNEL and cleaved caspase 3 staining was increased, while Proliferating Cell Nuclear Antigen (PCNA), an indicator for cell proliferation, was decreased in triptolidetreated group. Original amplification, 400×.

JNK1/2, and P38. We observed that the phosphorylation levels of these kinases were significantly increased by triptolide in a time- and concentration-dependent manner, without the corresponding alterations in term of total protein and mRNA 
A

B

\section{C}

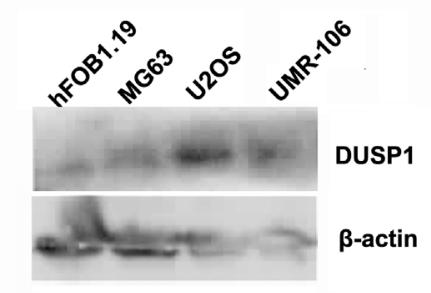

D

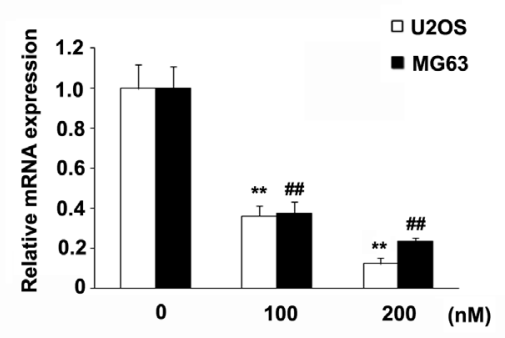

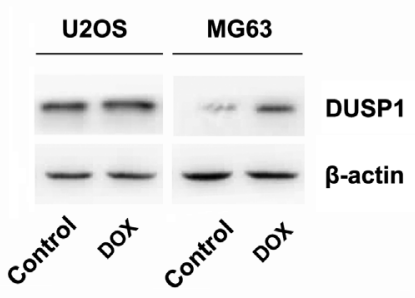

E
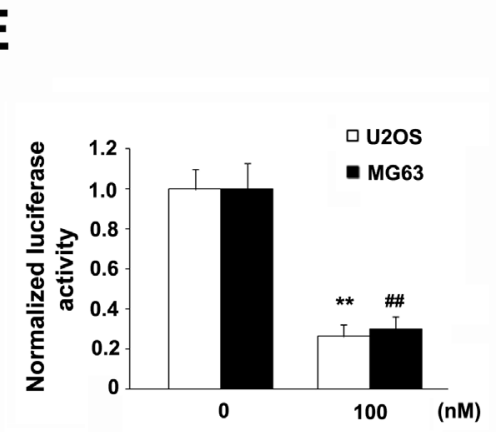

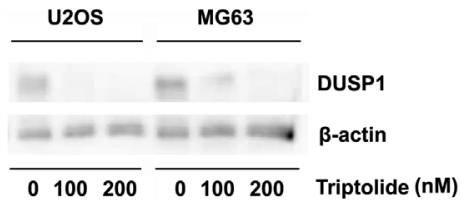

$\mathbf{F}$

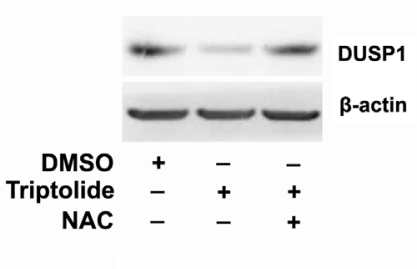

Figure 4. Effects of triptolide on DUSP1 expression in OS cells. (A) The basal protein expression of DUSP1 in three OS cells (MG63, U2OS, UMR-106) and osteoblast cell hFOB1.19 was measured by western blot. (B) Subtoxic dose of doxorubicin (DOX) increases DUSP1 levels in U2OS and MG63 cells. These cells were treated with doxorubicin $(0.1 \mathrm{ug} / \mathrm{ml})$ for 3 days, and then total proteins were subjected to western blot analysis. (C) Triptolide inhibits protein levels of DUSP1 in U2OS and MG63 cells; cells were treated with indicated concentrations of triptolide for $24 \mathrm{~h}$, then total proteins were extracted and used for western blot analysis. (D) Triptolide decreases mRNA expression of DUSP1 in U2OS and MG63 cells; cells were incubated with indicated concentrations of triptolide for $12 \mathrm{~h}$, then total RNA was extracted using TRIzol reagent, and qRT-PCR was performed to quantify the mRNA expression of DUSP1. ${ }^{* *} \mathrm{p}<0.01,{ }^{* *} \mathrm{p}<0.01$. (E) Triptolide inhibits the promoter activity of DUSP1; U2OS cells seeded into 24 -well plates and cotransfected with pGL3-DUSP1-luc and pRL-TK vectors; the relative luciferase activity was normalized with renilla luciferase activity. Each experiment was performed in quadruplicate. Data are expressed as the mean \pm standard deviation (SD). ${ }^{* *} \mathbf{p}<0.01,{ }^{* *} p<0.01$. (F) N-acetylcysteine (NAC) blocked triptolide-induced inhibition of DUSP1 in U2OS cells. Cells were treated with triptolide (100 nM) alone or combined with NAC (10 mM) for $12 \mathrm{~h}$. The expression of DUSP1 was measured by western blot.

levels (Figure 6A-C). Thus, we speculated whether the up-regulation of phosphorylated MAPKs could be attributed to decreased dephosphorylation mediated by DUSP1. To test this hypothesis, the effect of DUSP1 knockdown by siRNA on MAPKs phosphorylation was examined. Consistent with previous studies showing that DUSP1 dephosphorylates all these kinases, silencing of DUSP1 increased the phosphorylation levels of these MAPKs (Figure 6D). Considering that other MAPK phosphatases, such as DUSP2, DUSP4, and DUSP6, are also responsible for the dephosphorylation of these MAPKs $[25,10]$, we wondered whether the inhibition of DUSP1 was indispensable for the triptolide-induced phosphorylation of these kinases. As shown in Figure 6E, ectopic overexpression of DUSP1 in U2OS cells counteracted triptolide-induced phosphorylation of these MAPKs, suggesting that these alterations are DUSP1-dependent. The triptolide-induced modulation of MAPKs has been reported. However, the biological effects of this alteration in cancer cells remain controversial [26]. Thus, the inhibitors of
MAPKs, PD98059, SB203580, and SP600125 were employed to further investigate the role of the MAPKs in the antitumor effect of triptolide in OS cells. As shown in Figure 6F, triptolide-induced apoptosis was partially blocked by JNK inhibitor SP600125 and ERK inhibitor PD98059 but not by P38 inhibitor SB203580. Interestingly, similar results were observed when all these inhibitors were co-incubated with triptolide in OS cells (Figure 6G). Briefly, triptolide increased the phosphorylation of three major subfamilies of MAPKs through inhibiting DUSP1, which enhanced the triptolideinduced apoptosis in OS cells.

\section{Discussion}

Osteosarcoma (OS) is generally fatal due to its resistance to chemotherapies and lung metastasis. In the present study, we demonstrated that triptolide possesses potent and multidimensional antitumor activities in OS cells, encompassing the significant inhibition of cell viability, migration, invasion, 
A

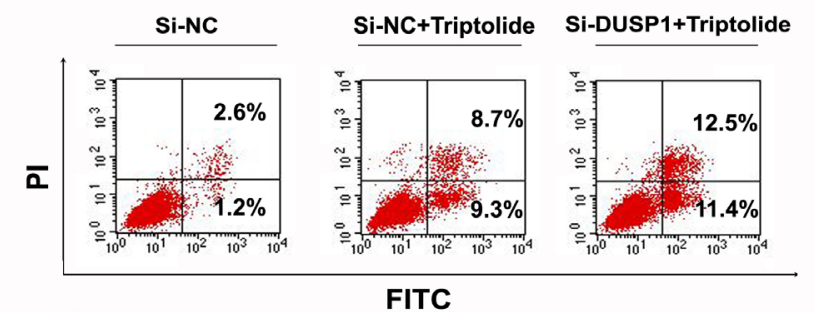

C

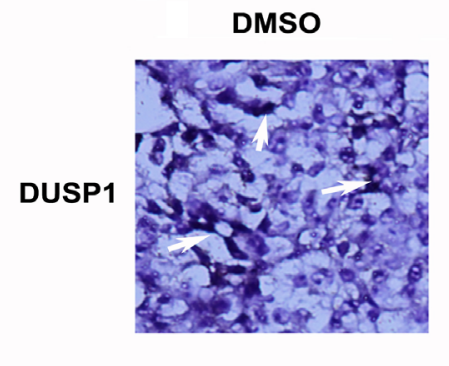

$\mathbf{E}$

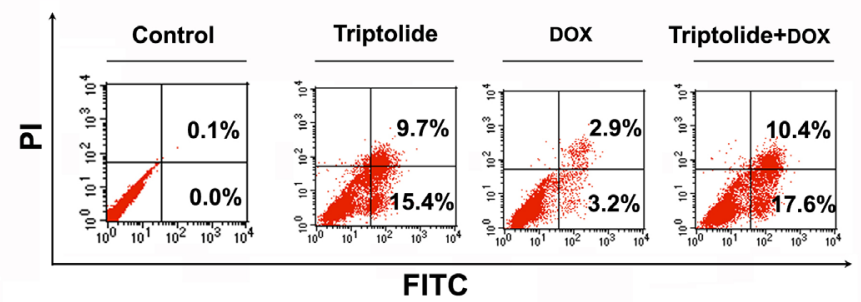

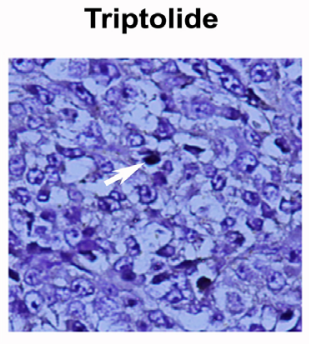

$\mathbf{F}$
B

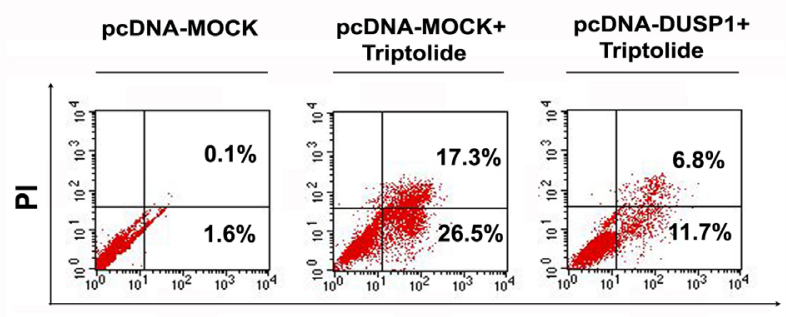

FITC

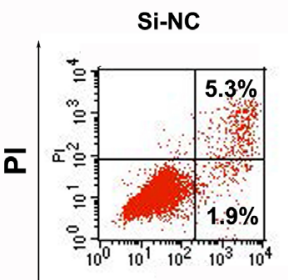

Si-NC+DOX

Si-DUSP1+DOX
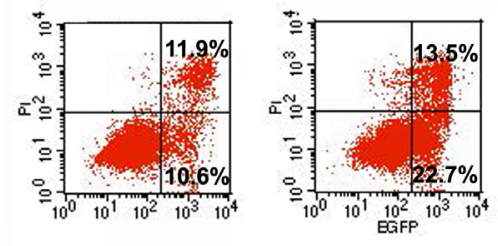

FITC

$\mathbf{F}$

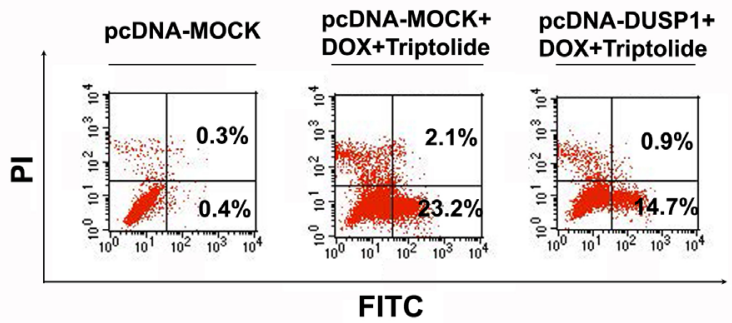

Figure 5. Inhibition of DUSP1 contributes to triptolide-induced apoptosis in OS cells. Representative FACS data are shown. (A) Knockdown expression of DUSP1 using specific siRNA (si-DUSP1) promotes triptolide-induced apoptosis; U2OS cells were transfected with si-DUSP1 for $6 \mathrm{~h}$, then triptolide was added into culture medium; cell apoptosis was measured by FACS. Nonspecific siRNA (si-NC) served as a negative control. (B) Ectopic over-expression of DUSP1 rescues U2OS cells from triptolide-induced apoptosis; cells transfected with pcDNA-MOCK or pcDNA-DUSP1 were treated with triptolide (100 nM) for $24 \mathrm{~h}$; apoptotic cells were detected by FACS. Cells transfected with pcDNA-MOCK alone served as a negative control. (C) The treatment of triptolide decreased DUSP1 expression in xenografted tumors. (D) Silencing of DUSP1 using specific siRNA (si-DUSP1) promotes doxorubicin-induced apoptosis. (E) Triptolide enhances doxorubicin-induced apoptosis in U2OS cells; cells were treated with triptolide (100 nM) and doxorubicin $(5 \mathrm{ug} / \mathrm{ml})$, alone or combined, for $24 \mathrm{~h}$; cell apoptosis was assessed by FACS. (F) Ectopic overexpression of DUSP1 partially counteracts the synergistic effects between triptolide and doxorubicin. U2OS cells transfected with pcDNA-MOCK or pcDNA-DUSP1 were co-incubated with triptolide and doxorubicin for $24 \mathrm{~h}$, then apoptotic cells were detected by FACS.

and cell cycle progression, as well as the induction of P53-independent mitochondrial apoptosis. Furthermore, the in vivo antitumor effects were also verified, as evidenced by the decreased xenograft tumor growth and lung metastasis in triptolide-treated mice. Thus, the multifactorial anticancer effects of triptolide make it an interesting candidate as a potential therapeutic agent for OS treatment.

DUSP1 is one of the major negative regulators of MAPKs, which are involved in the regulation of cell death and homeostasis in response to external stimuli. Abnormalities in DUSP1/MAPKs signaling pathways have been implicated in a wide range of human malignancies. DUSP1 is up-regulated in a wide range of human cancers, resulting in aberrant MAPKs pathways activity [9]. Moreover, because of the key role these MAPKs pathways play in mediating stressinduced apoptosis, the up-regulation of DUSP1 positively correlates with resistance of cancer cells to chemotherapies which rely on JNK- or p38-induced apoptosis $[27,10]$. 

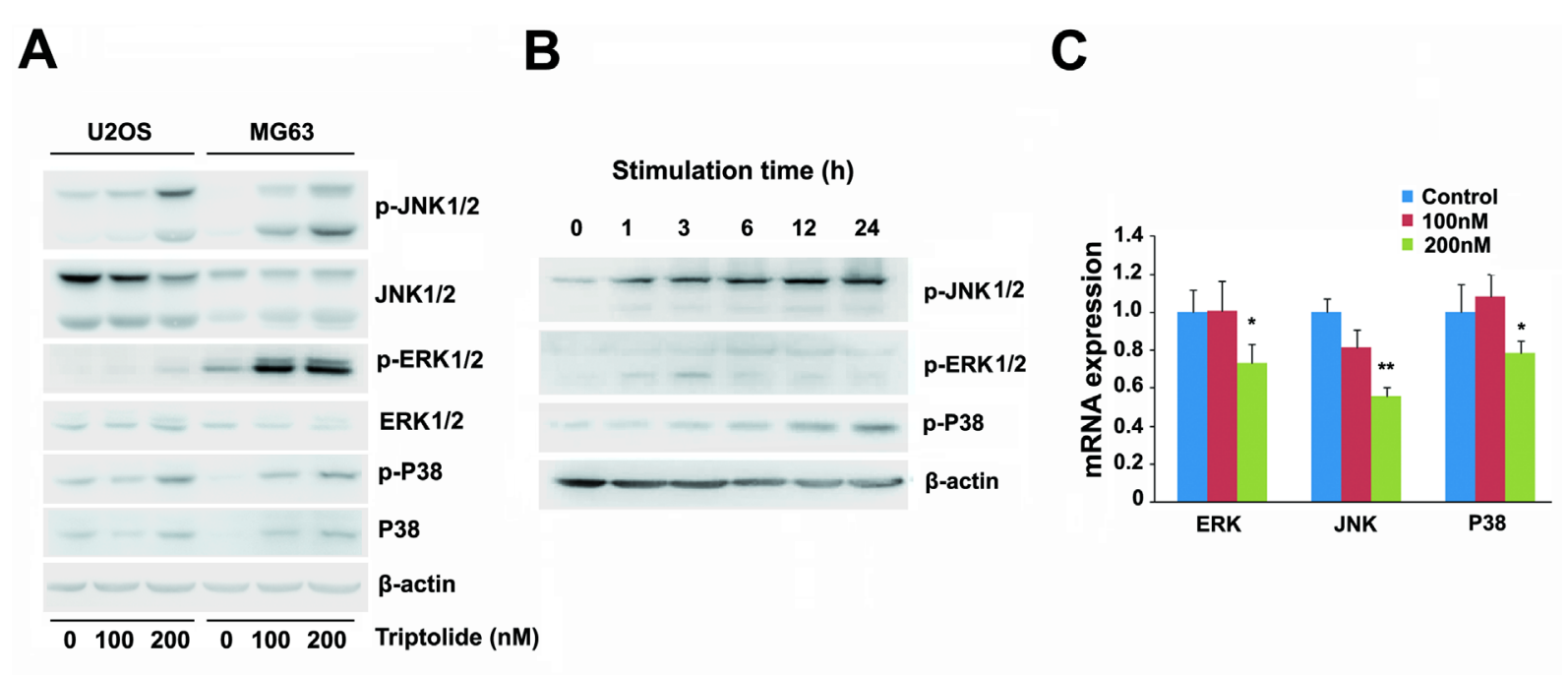

D

E
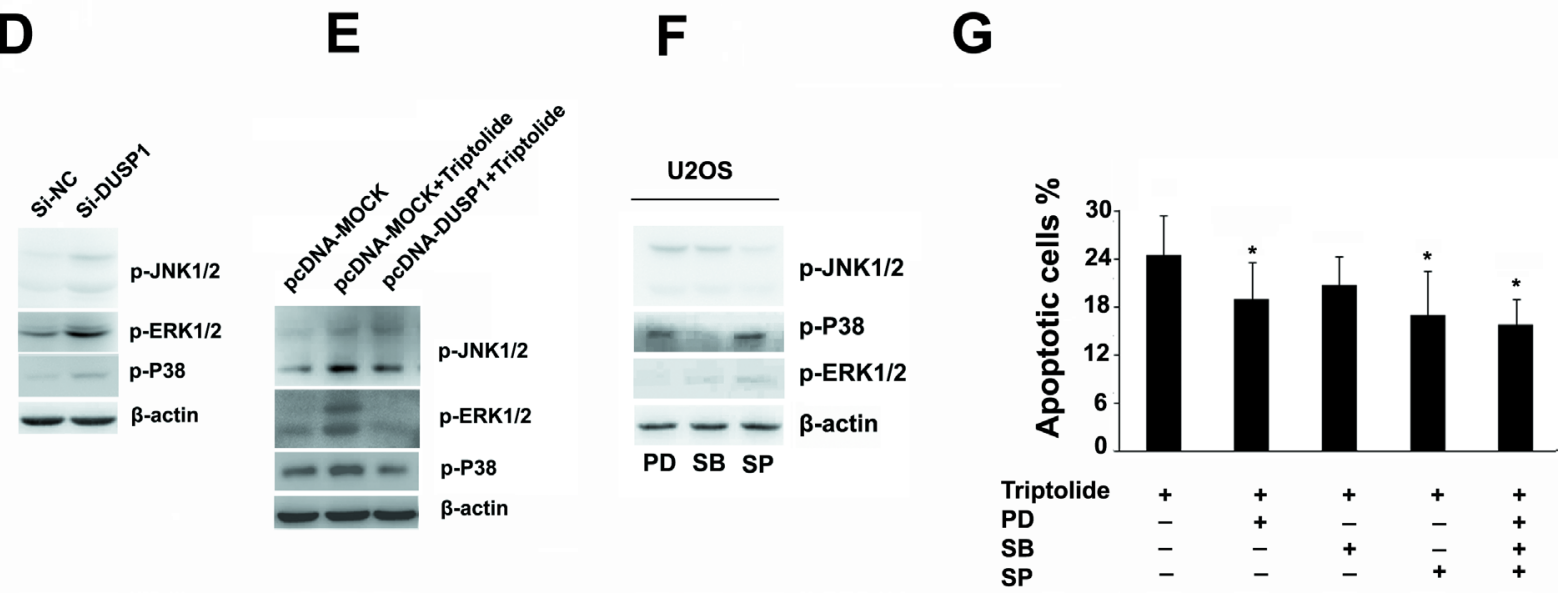

Figure 6. The effects of triptolide on MAPKs pathway. (A) The expression of p-JNK1/2, JNK1/2, p-ERK1/2, ERK1/2, p-P38, and P38 in triptolide-treated OS cells; U2OS and MG63 cells were treated with indicated concentrations of triptolide for $24 \mathrm{~h}$, then total proteins were extracted for western blot analysis. (B) Triptolide increases phosphorylation level of MAPKs; U2OS cells were exposed to $100 \mathrm{nM}$ triptolide for indicated time points, phosphorylated ERK, JNK and P38 MAPKs were measured by western blot. (C) The effects of triptolide on mRNA expression of MAPKs; U2OS cells were incubated with indicated concentrations of triptolide for $12 \mathrm{~h}$, then total RNA was extracted for subsequent qRT-PCR analysis. (D) The silencing of DUSP1 using si-RNA increases phosphorylated MAPKs. U2OS cells were transfected with si-DUSP1 or si-NC for $48 \mathrm{~h}$, and then protein samples were analyzed by western blot. (E) Ectopic over-expression of DUSP1 counteracts triptolide-induced phosphorylation of MAPKs. U2OS cells were transfected with pcDNA-DUSP1 or pcDNA-MOCK. After incubation for $24 \mathrm{~h}$, these cells were incubated with triptolide for additional $24 \mathrm{~h}$. The phosphorylated MAPKs were measured by western blot. (F) Effects of specific inhibitors on the phosphorylation of MAPKs. Cells were treated with ERK inhibitor PD98059 (PD, $50 \mathrm{uM}$ ), p38 inhibitor SB203580 (SB, $25 \mathrm{uM}$ ), and JNK1/2 inhibitor SP 600125 (SP, $25 \mathrm{uM}$ ) for $6 \mathrm{~h}$, then total proteins were extracted and subjected to western blot analysis. (G) The effects of MAPKs specific inhibitors on triptolide-induced apoptosis in OS cells. Cells were pretreated with PD, SB, and $\mathrm{SP}$ for $\mathbf{2} \mathrm{h}$, alone or combined. After exposure to $100 \mathrm{nM}$ triptolide for additional $24 \mathrm{~h}$, these cells were collected for apoptosis assay. ${ }^{\star} \mathrm{p}<0.005$.

The active role of DUSP1 in both tumor tumorigenesis and tumor resistance to therapies makes this enzyme a potential target for inhibitory drugs in cancer therapy. However, besides the oncogenic roles in human malignancies, DUSP1 may also function as a tumor suppressor, as overexpressed DUSP1 has been shown to down-regulate ras-dependent mitogenic signals $[28,29]$, and ectopic overexpression of DUSP1 reduced cell proliferation, invasiveness, and migration in cancer cells [30]. The putative tumor suppressor function of DUSP1 in certain tumor types or tumor stages may make the use of DUSP inhibitors non beneficial or even harmful in some cases, thus adding a new layer of complexity in DUSP1-targetd cancer therapy.

Triptolide is generally considered as a multi-target natural product, and a couple of signaling molecules including DUSP1 have been suggested to be its cellular targets [13]. It is reported that triptolide potently blocks the induction of DUSP-1 induced by peptidoglycan and activates pro-inflam- 
matory signaling [18]. However, the effect and mechanism of triptolide on the expression of DUSP1 in cancer cells remains largely unknown. Here we demonstrated that triptolide suppressed the promoter activity of DUSP1, leading to subsequent transcriptional and translational inhibition. It was reported that ROS accumulation could cause oxidation and inhibition of DUSP1 by converting its catalytic cysteine to sulfenic acid [24]. We demonstrated that triptolide potently increases the ROS production and consequently causes further destabilization of DUSP1, leading to sustained phosphorylation of all MAPKs subfamilies, including ERK1/2, JNK1/2, and P38. Importantly, the modulation of DUSP1/MAPKs cascade contributed to triptolide-induced apoptosis, as both the ectopic overexpression of DUSP1 and the knockdown of MAPKs using specific inhibitors could partially antagonize triptolide-mediated apoptosis. Specifically, the increased phosphorylation of JNK and ERK was proven to contribute to triptolide-induced apoptosis, since induction of apoptosis was blocked by the ERK inhibitor PD98059 and the JNK inhibitor SP600125 but not by the p38 MAPK inhibitor SB203580.

MAPKs are signaling components that are vital in converting extracellular stimuli into a wide range of cellular responses. ERKs generally exhibit pro-survival activities in tumors, while $\mathrm{p} 38 \mathrm{~s}$ and JNKs are major pro-apoptotic regulators. Thus, it makes sense that the activation of JNK contributes to triptolide-induced apoptosis. It is well established that transient activation of ERK1/2 can promote cell survival, mainly by promoting the activity of antiapoptotic proteins, such as Bcl-2, Mcl-1, and repressing proapoptotic proteins, such as Bad and Bim [31]. However, depending on the cell type and stimulus, ERK activity may also mediate anti-proliferative events. Increasing number of studies has shown that sustained ERK activation can promote intrinsic apoptotic pathways by induction of mitochondrial cytochrome $\mathrm{c}$ release and caspase-9 activation [28]. Actually, the proapoptotic function of ERK has been widely implicated in apoptosis induced by DNA-damaging agents, such as cisplatin, etoposide, and doxorubicin [32-34]. In this study, we also observed a sustained phosphorylation of ERK1/2, accompanied by increased mitochondrial cytochrome c release, Bax / Bcl-2 ratios, and caspase- 9 activation (Figure 2). Thus, ERK probably functions as a proapoptotic regulator in triptolidetreated OS cells.

Although the modulation of MAPKs by triptolide has been previously observed, the underlying mechanism and its biological effects seem elusive and contradictory. It was shown that triptolide exerts its neuroprotective effects via inhibiting the phosphorylation of p38, ERK1/2 [35]. Similarly, triptolide can inhibit the phosphorylation of JNK and p38 in C57BL/6 mice, which contributes to the decreased hepatic injury induced by lipopolysaccharide and D-galactosamine [36]. It was suggested that triptolide can also increase the phosphorylation of MAPKs in several cancer types; however, the question of whether the activation of MAPKs is involved in the antitumor activities of triptolide remains controversial. For example, it was reported that triptolide inhibited the growth of immortalized HT22 hippocampal cells via persistent activation of ERK-1/2 [37]. Conversely, Li et al. demonstrated that MAPKs were not involved in triptolideinduced cell growth inhibition and apoptosis in prostate cancer cell lines [26]. In the present study, we observed that triptolide simultaneously and stably activated these kinases in a DUSP1-denpendent manner, which in turn contributed to triptolide-induced apoptosis. Noticeably, DUSP1 was aberrantly up-regulated in OS cells, resulting in the inactivation of MAPKs. Thus, we speculated that the dual-directional modulation of MAPKs induced by triptolide was contextand cellular-dependent, and the basal expression levels of DUSP1 could be a key factor which determines the cellular responses to triptolide.

\section{Conclusion}

Finally, we demonstrated that triptolide-mediated inhibition of DUSP1 lead to sustained activation of MAPKs pathways, which in turn enhances triptolide-induced apoptosis and sensitizes OS cells to doxorubicin-induced cell death. The potent and broad antitumor activities of triptolide make it a potential therapeutic agent for the treatment of OS patients.

Acknowledgements: We thank our lab colleagues for helpful technical suggestions and critically discussing our data. This study was supported by National Natural Science Foundation of China (Grant Numbers 81073106 and 81202866) and Shanghai Municipal Science and Technology Commission (Grant Numbers 15ZR1414300).

\section{References}

[1] DAI X, MA W, HE X, JHA RK. Review of therapeutic strategies for osteosarcoma, chondrosarcoma, and Ewing's sarcoma. Med Sci Monit 2011; 17: RA177-190.

[2] HAMEED M, DORFMAN H. Primary malignant bone tumors--recent developments. Semin Diagn Pathol 2011; 28: 86-101.

[3] FERGUSON WS, GOORIN AM. Current treatment of osteosarcoma. Cancer Invest 2001; 19: 292-315.

[4] KESHET Y, SEGER R. The MAP kinase signaling cascades: a system of hundreds of components regulates a diverse array of physiological functions. Methods Mol Biol 2010; 661: 3-38. doi: 10.1007/978-1-60761-795-2 1

[5] DHILLON AS, HAGAN S, RATH O, KOLCH W. MAP kinase signalling pathways in cancer. Oncogene 2007; 26: 3279-3290.

[6] WAGNER EF, NEBREDA AR. Signal integration by JNK and p38 MAPK pathways in cancer development. Nat Rev Cancer 2009; 9: 537-549. doi: 10.1038/nrc2694 
[7] DAVIS RJ. Signal transduction by the JNK group of MAP kinases. Cell 2000; 103: 239-252.

[8] ABRAHAM SM, CLARK AR. Dual-specificity phosphatase 1: a critical regulator of innate immune responses. Biochem Soc Trans 2006; 34 (Pt 6): 1018-1023.

[9] KEYSE SM. Dual-specificity MAP kinase phosphatases (MKPs) and cancer. Cancer Metastasis Rev 2008; 27: 253261. doi: $10.1007 / \mathrm{s} 10555-008-9123-1$

[10] NUNES-XAVIER C, ROMA-MATEO C, RIOS P, TARREGA C, CEJUDO-MARIN R et al. Dual-specificity MAP kinase phosphatases as targets of cancer treatment. Anticancer Agents Med Chem 2011; 11: 109-132.

[11] BOUTROS T, CHEVET E, METRAKOS P. Mitogen-activated protein (MAP) Kinase/MAP kinase phosphatase regulation: Roles in cell growth, death, and cancer. Pharmacol Rev 2008; 60: 261-310. doi: 10.1124/pr.107.00106

[12] WANG ZH, ZHOU JY, KANAKAPALLI D, BUCK S, WU GS et al. High Level of Mitogen-Activated Protein Kinase Phosphatase-1 Expression Is Associated With Cisplatin Resistance in Osteosarcoma. Pediatr Blood Cancer 2008; 51: 754-759. doi: $10.1002 / \mathrm{pbc} .21727$

[13] LIU Q. Triptolide and its expanding multiple pharmacological functions. Int Immunopharmacol. 2011; 11: 377-383. doi: 10.1016/j.intimp.2011.01.01

[14] WONG KF, YUAN Y, LUK JM. Tripterygium wilfordii bioactive compounds as anticancer and anti-inflammatory agents. Clin Exp Pharmacol Physiol 2012; 39: 311-320. doi: 10.1111/j.1440-1681.2011.05586.x

[15] LIU Z, MA L, ZHOU GB. The main anticancer bullets of the Chinese medicinal herb, thunder god vine. Molecules 2011; 16: 5283-5297. doi: 10.3390/molecules16065283

[16] ZHOU ZL, YANG YX, DING J, LI YC, MIAO ZH. Triptolide: structural modifications, structure-activity relationships, bioactivities, clinical development and mechanisms. Nat Prod Rep 2012; 29: 457-475. doi: 10.1039/c2np00088a

[17] PAN J. RNA polymerase - an important molecular target of triptolide in cancer cells. Cancer Lett 2010; 292: 149-152. doi: 10.1016/j.canlet.2009.11.018

[18] SHEPHERD EG, ZHAO Q, WELTY SE, HANSEN TN, SMITH CV et al. The function of mitogen-activated protein kinase phosphatase- 1 in peptidoglycan-stimulated macrophages. J Biol Chem 2004; 279: 54023-54031.

[19] LIU YX, WANG JL, GUO JF, WU JJ, LIEBERMAN HB et al. DUSP1 is controlled by p53 during the cellular response to oxidative stress. Mol Cancer Res 2008; 6: 624-633. doi: 10.1158/1541-7786.MCR-07-2019

[20] FISHER JL, MACKIE PS, HOWARD ML, ZHOU H, CHOONG PF. The expression of the urokinase plasminogen activator system in metastatic murine osteosarcoma: an in vivo mouse model. Clin Cancer Res 2001; 7: 1654-1660.

[21] JIANG XH, WONG BC, LIN MC, ZHU GH, KUNG HF et al. Functional p53 is required for triptolide-induced apoptosis and AP-1 and nuclear factor-kappaB activation in gastric cancer cells. Oncogene 2001; 20: 8009-8018. doi: 10.1038/ sj.onc. 1204981
[22] RAY PD, HUANG BW, TSUJI Y. Reactive oxygen species (ROS) homeostasis and redox regulation in cellular signaling. Cell Signal 2012; 24: 981-990. doi: 10.1016/j.cellsig.2012.01.008

[23] CHEN P, LI J, BARNES J, KOKKONEN GC, LEE JC et al. Restraint of proinflammatory cytokine biosynthesis by mitogen-activated protein kinase phosphatase-1 in lipopolysaccharide-stimulated macrophages. J Immunol 2002; 169: 6408-6416.

[24] KAMATA H, HONDA S-I, MAEDA S, CHANG L, HIRATA $H$ et al. Reactive Oxygen Species Promote TNFaInduced Death and Sustained JNK Activation by Inhibiting MAP Kinase Phosphatases. Cell 2005; 120: 649-661.

[25] PATTERSON KI, BRUMMER T, O'BRIEN PM, DALY RJ. Dual-specificity phosphatases: critical regulators with diverse cellular targets. Biochem J 2009; 418: 475-489.

[26] LI W, LIU Y, LI XX, YU Y, WU JJ et al. MAPKs are not involved in triptolide-induced cell growth inhibition and apoptosis in prostate cancer cell lines with different p53 status. Planta Med 2011; 77: 27-31. doi: 10.1055/s-0030-1250076

[27] HAAGENSON KK, WU GS. The role of MAP kinases and MAP kinase phosphatase-1 in resistance to breast cancer treatment. Cancer Metastasis Rev 2010; 29: 143-149. doi: 10.1007/s10555-010-9208-5

[28] MARSHALL CJ. Specificity of receptor tyrosine kinase signaling: transient versus sustained extracellular signal-regulated kinase activation. Cell 1995; 80: 179-185.

[29] MANZANO RG, MONTUENGA LM, DAYTON M, DENT $\mathrm{P}$, KINOSHITA I et al. CL100 expression is down-regulated in advanced epithelial ovarian cancer and its re-expression decreasesitsmalignant potential. Oncogene2002;21:4435-4447.

[30] TAI CJ, WU AT, CHIOU JF, JAN HJ, WEI HJ et al. The investigation of mitogen-activated protein kinase phosphatase- 1 as a potential pharmacological target in non-small cell lung carcinomas, assisted by non-invasive molecular imaging. BMC Cancer 2010; 10: 95. doi: 10.1186/1471-2407-10-95

[31] BALMANNO K, COOK SJ. Tumour cell survival signalling by the ERK1/2 pathway. Cell Death Differ 2009; 16: 368-377. doi: $10.1038 /$ cdd. 2008.148

[32] WANG X, MARTINDALE JL, HOLBROOK NJ. Requirement for ERK activation in cisplatin-induced apoptosis. J Biol Chem 2000; 275: 39435-3943.

[33] STEFANELLI C, TANTINI B, FATTORI M, STANIC I, PIGNATTI $C$ et al. Caspase activation in etoposide-treated fibroblasts is correlated to ERK phosphorylation and both events are blocked by polyamine depletion. FEBS Lett 2002; 527: 223-228.

[34] YEH PY, CHUANG SE, YEH KH, SONG YC, CHANG LL et al. Phosphorylation of $\mathrm{p} 53$ on Thr55 by ERK2 is necessary for doxorubicin-induced p53 activation and cell death. Oncogene 2004; 23: 3580-3588.

[35] GENG Y, FANG M, WANG J, YU H, HU Z et al. Triptolide down-regulates COX-2 expression and PGE2 release by suppressing the activity of NF-kappaB and MAP kinases in lipopolysaccharide-treated PC12 cells. Phytother Res 2012; 26: $337-343$. doi: $10.1002 /$ ptr.3538 
[36] LU Y, BAO X, SUN T, XU J, ZHENG W et al. Triptolide attenuate the oxidative stress induced by LPS/D-GalN in mice. J Cell Biochem 2012; 113: 1022-1033. doi: 10.1002/jcb.23434

[37] KOO HS, KANG SD, LEE JH, KIM NH, CHUNG HT et al. Triptolide Inhibits the Proliferation of Immortalized HT22
Hippocampal Cells Via Persistent Activation of Extracellular Signal-Regulated Kinase-1/2 by Down-Regulating Mitogen-Activated Protein Kinase Phosphatase-1 Expression. J Korean Neurosurg Soc 2009; 46: 389-396. doi: 10.3340/ jkns.2009.46.4.389 